\title{
Improved Probability Algorithm Based on Low Energy Bluetooth Technology
}

\author{
Lin Zhu \\ Guiyang University, Guiyang, 550005, China \\ byronalder@hotmail.com
}

Keywords: Location Fingerprints, Bluetooth Low Energy, Distribution, Probabilistic Algorithms

\begin{abstract}
With the use of low energy Bluetooth technology, it aims to examine the positioning accuracy and performance of location fingerprint algorithm and thus to make improvements. To locate by means of Bluetooth 4.0 (i.e. low-energy Bluetooth tech) is energy saving, costing less, deploying easily etc. So here we choose Bluetooth 4.0 as the positioning way to make in-depth studies. Besides, we choose location fingerprint positioning method for the reason that the method has many merits like easy implementation, low costs, quick creation of correct mathematical model. It can be effectively and quickly put into practical applications.
\end{abstract}

\section{Introduction}

The positioning algorithm, as the core to indoor positioning system, is decisive to the system's positioning accuracy [1-2]. Bluetooth-based positioning method was developed when the indoor localization just began. The indoor positioning chip BCM4752 developed by Broadcom provides simultaneously several technologies like WLAN [3], Bluetooth and NFC as to support the indoor localization. With the release of BLE technology of Bluetooth 4.0, the Bluetooth application finally shows its own features in the field of indoor positioning [4].

Bluetooth AP cost is lower than WLAN. Moreover, in real practice, the deployment of low-energy Bluetooth is relatively simple, because such Bluetooth AP does not require power supply from the external power source; instead, one button cell can be enough to supply power for over a year. Also, with the Bluetooth positioning way, it's not necessary to install any additional devices in the mobile phone end, with favorably higher positioning accuracy [5].

Comparing with other methods, the location fingerprint positioning method makes full use of this feature, signal multi-path broadcast, which is caused by indoor environment complications, declining in some extent the number of positioning base stations to be deployed. Also different from the path loss method and other positioning methods imported by outdoor positioning, the proposed method can reach better positioning accuracy. The obvious effect of too complicated indoor environments on signal multi-path broadcast is an intractable issue to other related methods. But the location fingerprint utilizes well the shortcoming for positioning, to a large extent reducing the indoor positioning errors. That's why we take the method for positioning.

\section{Improvement of the probabilistic algorithm in applications}

Probabilistic algorithm is one of the important positioning methods in the location fingerprint positioning. Considering RSSI values of each position may not be stable [6-7], we introduce the probabilistic mechanism in the statistics to the positioning to form one type of positioning method based on probability. We do so in order to show truly the variation of RSSI values in different locations for better positioning precision. With reference to the analysis of RSSI distribution characteristics, we attempt to upgrade the application of probabilistic algorithm in the indoor positioning system based on low energy Bluetooth.

Conventional probability methods. Among the probability methods, naive Bayes method is representative. It derived from Bayes classification, which is a statistical classifying method. Unlike 
deterministic methods, probabilistic ones can only give the probability at which a real-time sampling signal belongs to one reference point rather than telling positively it belongs to which one. The process of positioning by naive Bayes method includes two periods: (1) offline period: the probabilistic method needs to store RSSI values of each reference point from different AP samples and the probability how often the point comes up as to create fingerprint database; hence, the storage space should be bigger than deterministic methods; (2) real-time positioning period: the user can scan the position at its mobile client end to get the mean value of RSSI samples from each AP; then according to Bayes theorem, the posterior probability can be estimated how often the RSSI sample appears in each reference point; next, one or more reference points with higher posterior probability are chosen to evaluate the location of the user.

Probability methods based on Bayes theorem and Gaussian distribution can decrease remarkably the sample amount of acquired data in each reference point in the offline period, thus to reduce the storage space. But from the analysis of RSSI distribution features, we learn that under the influence of surrounding environments, such features in most locations are not utterly consistent with Gaussian distribution. For that problem, lots of literatures proposed to solve it, for instance, the polynomial method for fitting, which requires plentiful computation work if it hopes to get more accurate positioning.

Improvement of the probabilistic method in applications. With regards to the probability method based on Gaussian distribution model, it doesn't require the fingerprint database, different from the conventional simple positioning methods. However, in actual cases, the Gaussian model can't fully adapt, which would affect the positioning accuracy. Therefore, we incorporate multiple matching models to cater for different positioning needs.

First of all, we introduce the positioning method based on Maxwell distribution model. The model was initially used as velocity-rate model in the physical science. But due to right avertence, we apply it in the positioning method. The probability density function of the model is like equation (1):

$$
f(l)=\sqrt{\frac{2}{\pi} \frac{l^{2} e^{-x^{2} /\left(2 a^{2}\right)}}{a^{3}}}
$$

Secondly, we use the least square method to get sample's probability function through fitting of samples acquired by the polynomial $f(x)=a_{0}+a_{1} x+\ldots+a_{n} x^{n}$. Also with the help of the least square method, we can consider taking exponential function to achieve the matching of sample's probabilistic function. The fitting principle of the least square method is to make minimum the quadratic sum $\delta^{2}$ of errands between fitting function $f(x)$ and sample value y, as shown in (2):

$$
\delta^{2}=\sum_{i=0}^{N}\left(f\left(x_{i}\right)-y_{i}\right)^{2}
$$

Finally, in the offline stage, we let sample mean value, standard deviation and skewness coefficient as measurement indices. Then, we build Gaussian distribution model, Maxwell model and polynomial model after RSSI values from each AP are acquired in every single reference point $x_{i}$. In the real-time period, different matching methods are used to calculate the value of probability for different samples.

\section{Experimentation and Results}

To validate the effect of the above positioning methods, we chose the place where we analyzed Bluetooth RSSI distribution features to perform emulation experiment, as shown in Fig. 1. The place is the corridor in the seventh floor of the building. We selected 18 reference points as sampling points, in the separate distance of $0.5 \mathrm{~m}$. In the offline period, every reference point gathers RSSI samples from AP for 100times, at an interval is $1 \mathrm{~s}$.

To compare probabilistic methods, we used Gaussian distribution model and Maxwell distribution model to test the performance of positioning in different locations far from all APs. We picked up one reference point which is farther away from three APs as experimental point. We 
performed the positioning for 100 iterations in the point as to obtain the error. After that, we plotted one error probability statistical graph. See Fig. 2 for details.

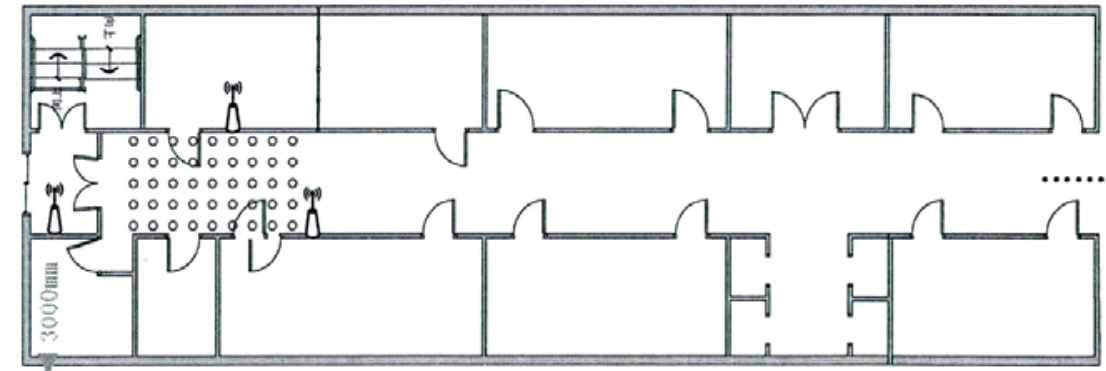

Figure1 the experimental site map of performance Analysis of the location algorithm

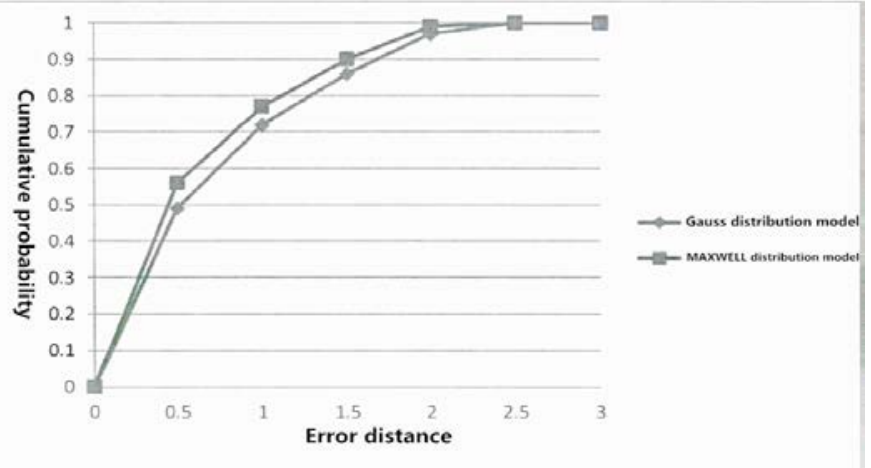

Figure2 Comparison of Gauss distribution model and MAXWELL distribution model

\section{Conclusions}

With view to the improvement of probabilistic method, we found when different models were built in different reference points, different matching methods were utilized for positioning, which is helpful to increase positioning accuracy. Also compared with the methods all by polynomial model matching, the computational work was declined when positioning was on, speeding up the positioning. So it's advisable to take the improved location fingerprint algorithm based on multiple models. It improves the precision and also reduces plentiful calculation amount involved with the local precise orientation algorithms.

\section{References}

[1] Wang Saiwei. WLAN indoor positioning method based on Finger. Harbin Institute of Technology (2009)

[2]Liu Wen, Xu Lianming, Li Zhifeng, Deng Zhongliang. Spatial grid based matching location algorithm. Journal of Huazhong University of Science and Technology (NATURAL SCIENCE EDITION), 2014, 03:19-22.

[3] Feng Yan, an indoor location method based on fingerprint. Science and technology information, 2014, 08: 17+20.

[4] Lie theory, Ding Enjie, Hao Lina, Zhang Lei. Matching algorithm is an improved coal mine location fingerprint. Journal of transducer technology, 2014, 03:388-393.

[5] Wang Zhongmin, Chen Zhen, Pan Chunhua. An indoor location algorithm of intelligent mobile phone location fingerprint improvement. Journal of Xi'an University of Posts and telecommunications, 2014, 01:17-20.

[6] Li Feng, a RSSI positioning method based on region partition. The application of computer system, 2014, 07:152-155.

[7] Wang Yanke, Lu Chuan. WAVE protocol stack of wireless location based on Gauss mixture model. Wireless Internet technology, 2014, 04:121-123. 